\title{
Effects of FHIT gene on proliferation and apoptosis of osteosarcoma cells
}

\author{
ZHENGFENG XU, JIAJUN WU, PAN CAI, XIAOXIAO ZHOU, CUNGUO YI and BIN WANG \\ Department of Orthopedics, Zhoupu Hospital Affiliated to Shanghai University of \\ Medicine and Health Science, Shanghai 201318, P.R. China
}

Received April 12, 2018; Accepted October 10, 2018

DOI: $10.3892 / \mathrm{ol} .2018 .9696$

\begin{abstract}
Regulatory effects of fragile histidine triad (FHIT) gene on proliferation and apoptosis of osteosarcoma cells were studied. The hFOB1.19 and Saos2 cells were routinely cultured, pcDNA3.1-FHIT overexpression vectors carrying FHIT gene fragments and blank pcDNA3.1 vectors were transfected into Saos 2 cells, respectively, and the cells were divided into hFOB, Saos2, transfection and no-load transfection groups. After transfection for $48 \mathrm{~h}$, the cells were collected and analyzed. The expression of FHIT messenger ribonucleic acid (mRNA) was detected by reverse transcription-quantitative polymerase chain reaction (RT-qPCR). The expression of FHIT protein was detected by western blot analysis. Cell Counting Kit 8 (CCK8) was used to detect cell proliferation, and flow cytometry was used to detect apoptosis. The expression of FHIT mRNA was significantly decreased in Saos2 group compared with that in hFOB group, and the difference was statistically significant $(\mathrm{P}<0.05)$. The expression of FHIT mRNA was significantly increased in transfection group compared with that in Saos2 group, and the difference was statistically significant $(\mathrm{P}<0.05)$. The expression of FHIT protein was obviously decreased in Saos 2 group compared with that in hFOB group, and there was a statistically significant difference $(\mathrm{P}<0.05)$. The expression of FHIT protein was obviously increased in transfection group compared with that in Saos2 group, and the difference was statistically significant $(\mathrm{P}<0.05)$. Compared with that in the hFOB group, the cell proliferation rate was remarkably increased in Saos 2 group, while the apoptosis rate was remarkably decreased, showing statistically significant differences $(\mathrm{P}<0.05)$. Compared with those in Saos2 group, the cell proliferation rate was significantly decreased in transfection group, while the apoptosis rate was significantly increased, and the differences were statistically significant $(\mathrm{P}<0.05)$. In
\end{abstract}

Correspondence to: Dr Bin Wang, Department of Orthopedics, Zhoupu Hospital Affiliated to Shanghai University of Medicine and Health Science, 1500 Zhouyuan Road, Shanghai 201318, P.R. China E-mail: wangbinxy2018@163.com

Key words: fragile histidine triad gene, osteosarcoma, cell proliferation, apoptosis conclusion, FHIT gene regulates the proliferation and apoptosis of Saos 2 osteosarcoma cells, inhibits the proliferation and promotes apoptosis of Saos2 osteosarcoma cells.

\section{Introduction}

Osteosarcoma is one of the most common clinical malignant tumors in orthopedics. It is a kind of primary bone tissue tumor with a high degree of malignancy, which often occurs in children and adolescents and has the characteristics of strong invasion, rapid metastasis and high mortality $(1,2)$. At present, there is no ideal treatment for osteosarcoma. The commonly used methods include surgery, radiotherapy and chemotherapy, but the curative effect is not good. According to statistics, the 5-year survival rate of osteosarcoma is only 50-60\% (3). Fragile histidine triad (FHIT) gene is an important member of histidine triad gene family. It is considered as a tumor suppressor gene, whose abnormal expression is associated with a variety of malignant tumors, such as lung, cancer and breast cancer. Studies have shown that $(4,5)$ FHIT gene plays an important regulatory role in the process of cell proliferation and apoptosis. However, the role of FHIT in osteosarcoma remains unclear. The purpose of the present study was to investigate the role of FHIT gene in the regulation of proliferation and apoptosis of Saos2 osteosarcoma cells, clarify its relationship with proliferation and apoptosis of osteosarcoma cells, and demonstrate that FHIT gene is as an effective target for the treatment of osteosarcoma.

\section{Materials and methods}

Materials and reagents. hFOB1.19 normal human osteoblastic cell line, Saos2 osteosarcoma cell line (both from American Type Culture Collection, Manassas, VA, USA), rabbit anti-epidermal growth factor receptor (EGFR), human estrogen receptor-2 (HER-2) monoclonal antibodies and secondary goat anti-rabbit polyclonal antibody (cat. nos. ab52894, ab134182, ab6721; Abcam, Cambridge, MA, USA), Roswell Park Memorial Institute (RPMI)-1640 medium, Dulbecco's modified Eagle's medium (DMEM-F12) (both from HyClone; GE Healthcare Life Sciences, Logan, UT, USA), fetal bovine serum (FBS), trypsin (both from Gibco; Thermo Fisher Scientific, Inc., Waltham, MA, USA), penicillin, streptomycin (both from Sigma-Aldrich; Merck KGaA, Darmstadt, 
Germany), Cell Counting Kit 8 (CCK8) (Beyotime Institute of Biotechnology, Hangzhou, China), AceQ reverse transcription-quantitative polymerase chain reaction (RT-qPCR) Synergy Brands (SYBR) Green Master Mix kit (Vazyme, Nanjing, China), HiScript II Q reverse transcription (RT) SuperMix for RT-qPCR [+genomic deoxyribonucleic acid (gDNA) wiper] kit (Vazyme), Annexin V-fluorescein isothiocyanate (FITC)/propidium iodide (PI) apoptosis detection kit (Beyotime Institute of Biotechnology), restriction endonuclease EcoRI and restriction endonuclease BamHI (both from Promega Corp., Madison, WI, USA).

The study was approved by the Ethics Committee of Zhoupu Hospital affiliated to Shanghai University of Medicine and Health Science (Shanghai, China).

Instruments. $\mathrm{CO}_{2}$ cell incubator (Thermo Fisher Scientific, Inc.), fluorescence microscope (Leica DMI 4000B/DFC425; Leica Microsystems GmbH, Wetzlar, Germany), NanoDrop ND-1000 spectrophotometer (NanoDrop Technology; Thermo Fisher Scientific, Inc., Wilmington, DE, USA), fluorescence RT-qPCR instrument (ABI 7500; Applied Biosystems, Foster City, CA, USA), microplate reader (Bio-Rad Laboratories, Inc., Hercules, CA, USA), FACS flow cytometer (BD Biosciences, Franklin Lakes, NJ, USA), Image Lab and Image-Pro image analysis systems (Bio-Rad Laboratories, Inc.).

Cell culture. hFOB1.19 cells were cultured in DMEM-F12 complete medium containing $10 \%$ FBS and $1 \%$ penicillin and streptomycin. Saos2 cells were cultured in RPMI complete medium containing 10\% FBS and 1\% penicillin and streptomycin. All cells were cultured in an incubator with $5 \% \mathrm{CO}_{2}$ at $37^{\circ} \mathrm{C}$, and the medium was changed once every 3 days, followed by passage when $80 \%$ of them were fused.

Cell passage. When $80 \%$ of the cultured cells were fused, the cell culture medium was removed, followed by digestion with $0.125 \%$ trypsin for $1 \mathrm{~min}$ and then gentle pipetting to digest the adherent cells. RIPM-1640 medium containing 10\% FBS was added to stop digestion, followed by centrifugation at 1,200 $\mathrm{xg}$ for $5 \mathrm{~min}$ at $4^{\circ} \mathrm{C}$. Then, the supernatant was removed, the medium was added for resuspension, and the cells continued to be cultured. Finally, the cells were collected for experiments when they were passaged to the third generation.

Cell treatment and transfection. Cells were divided into hFOB, Saos2, transfection and no-load transfection groups. hFOB cells were routinely cultured in hFOB group without any treatment. Saos2 cells were routinely cultured in Saos2 group without any treatment. pcDNA3.1-FHIT overexpression vectors containing FHIT gene were transfected into the cultured cells in the transfection group. pcDNA3.1 vectors were transfected into the cultured cells in the no-load transfection group.

Transfection methods: pcDNA3.1-FHIT overexpression vectors were constructed, followed by amplification of FHIT gene in hFOB1.9 cell line and identification of pMD10-T vector sequencing. pMD10-T recombinant vectors containing correct FHIT gene fragments were dually digested with EcoRI and BamHI, and cloned into pcDNA3.1 eukaryotic expression vectors. The overexpression plasmids containing correct sequences were mixed with liposomes, diluted with RPMI medium and allowed to rest at room temperature for $20 \mathrm{~min}$. Finally, the cells were implanted and cultured with cell culture medium for $48 \mathrm{~h}$.

Western blot analysis. After transfection, the cells in each group were collected with ProteoPrep ${ }^{\circledR}$ Total Extraction Sample kit (Sigma-Aldrich, Merck KGaA, Darmstadt, Germany) and added with lysis buffer, followed by ice bath for $60 \mathrm{~min}$ and centrifugation at $14,000 \mathrm{x}$ g for $10 \mathrm{~min}$ at $4^{\circ} \mathrm{C}$, and the protein was quantified by bicinchoninic acid (BCA) method. The standard curve and absorbance were measured by the microplate reader and the protein concentration was calculated. After denaturation of the protein, the samples were loaded (10 $\mu \mathrm{l}$ per lane) and separated by $15 \%$ sodium dodecyl sulfate-polyacrylamide gel electropheresis (SDS-PAGE) with corresponding concentration. When the marker protein ran to the bottom of the glass plate and the sample protein was basically in a straight line sinking to the bottom, the gel running was stopped. The samples were transferred onto a polyvinylidene fluoride (PVDF) membrane, sealed, rinsed with phosphate-buffered saline with Tween-20 (PBST) 3 times, and then sealed with sealing solution for $1.5 \mathrm{~h}$. Primary anti-human EGFR, HER-2 monoclonal antibodies $(1: 1,000)$ and secondary antibody $(1: 1,000)$ were added successively. The membrane was rinsed with Tris-buffered saline with Tween-20 (TBST) at each step interval. After being rinsed with TBST to remove the secondary antibody, color development began. The membrane was placed in the chemiluminescence reagent (Beyotime Institute of Biotechnology, Shanghai, China) for reaction for $1 \mathrm{~min}$ and then developed in dark conditions. Finally, gel scanning imaging system (Bio-Rad Laboratories, Inc.) was used for analysis.

RT-qPCR detection. Total RNA was extracted using TRIzol (Thermo Fisher Scientific, Inc.) from spare bone blocks stored at $-20^{\circ} \mathrm{C}$. The extracted total RNA was reversely transcribed into complementary DNA (cDNA) using RT kit (ABclonal Biotech Co., Ltd., Wuhan, China), and the reaction system was $20 \mu 1$. Fast SYBR Green Master Mix was used. The reaction conditions were as follows: reaction at $51^{\circ} \mathrm{C}$ for 2 min, predenaturation at $96^{\circ} \mathrm{C}$ for $10 \mathrm{~min}$, denaturation at $96^{\circ} \mathrm{C}$ for $10 \mathrm{sec}$, annealing at $60^{\circ} \mathrm{C}$ for $30 \mathrm{sec}, 40$ cycles. The relative expression of FHIT messenger ribonucleic acid (mRNA) was calculated. The results were analyzed by using the $2^{-\Delta \Delta \mathrm{Cq}}$ method (6). Primer sequences are shown in Table I.

Detection of cell proliferation by CCK8. After cell transfection, the cells in each group were inoculated into a 96-well plate at a density of $3 \times 10^{3} / \mathrm{ml}$. Three replicate wells were set and each well was added with $100 \mu 1$ of complete medium. The cells were cultured in an incubator with $5 \% \mathrm{CO}_{2}$ at $37^{\circ} \mathrm{C}$ for $24 \mathrm{~h}$. Each well was added with $10 \mu \mathrm{l}$ of CCK8 solution, followed by incubation under the above conditions for $1 \mathrm{~h}$. The optical density (OD) value was measured at a wavelength of $450 \mathrm{~nm}$ to calculate the cell proliferation rate.

Detection of apoptosis by flow cytometry. After cell transfection, the cells in each group were collected and rinsed with PBS, and then the supernatant was discarded. The cells were 
Table I. Primer sequences.

\begin{tabular}{ll}
\hline Name & \multicolumn{1}{c}{ Primer sequences } \\
\hline FHIT & F: 5'-TTGGGGCGCGGGTTTGGGTTTTTAC-3' \\
& R: 5'-CGTAAACGACGCCGACCCCACTA-3' \\
GAPDH & F: 5'-ACGGCAAGTTCAACGGCACAG-3' \\
& R: 5'-GAAGACGCCAGTAGACTCCACGAC-3'
\end{tabular}

FHIT, fragile histidine triad; GADPH, glyceraldehyde 3-phosphate dehydrogenase.

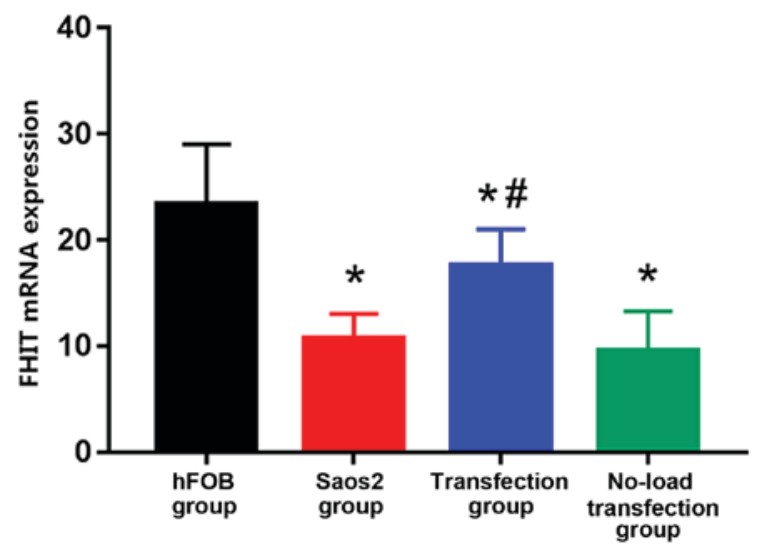

Figure 1. FHIT mRNA expression. " $\mathrm{P}<0.05$ vs. $\mathrm{hFOB}$ group; ${ }^{\text {}} \mathrm{P}<0.05$ vs. Saos2 group. FHIT, fragile histidine triad.

resuspended with binding buffer and the concentration was adjusted to $1 \times 10^{5} / \mathrm{ml}$. Annexin V-FITC solution $(10 \mu \mathrm{l})$ and $5 \mu \mathrm{l}$ PI solution were added and mixed well. After reaction in the dark at room temperature for $15 \mathrm{~min}$, the mixture was detected by flow cytometry. Data were analyzed by cytomics fc 500 (Beckman Coulter, Inc., Atlanta, GA, USA).

Statistical analysis. In this study, Statistical Product and Service Solutions (SPSS) 20.0 software (IBM Corp., Armonk, NY, USA) was used for statistical analysis. Enumeration data are presented as mean \pm standard deviation. t-test was used for data in line with the normal distribution and homogeneity of variance, corrected t-test was used for data in line with the normal distribution and heterogeneity of variance, and non-parametric test was used for data in line with the abnormal distribution and heterogeneity of variance. Chi-square test was used for enumeration data.

\section{Results}

Detection of FHIT mRNA expression via RT-qPCR. The expression of FHIT mRNA was the highest in hFOB group and the lowest in Saos2 group. Compared with that in hFOB group, the expression levels of FHIT mRNA in the other groups were significantly decreased, and the differences were statistically significant $(\mathrm{P}<0.05)$. Compared with that in Saos2 group, the expression of FHIT mRNA in transfection group was significantly increased, and the difference was statistically significant $(\mathrm{P}<0.05)$ (Fig. 1). The results suggested that

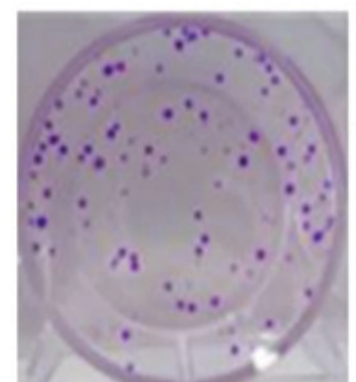

hFOB group

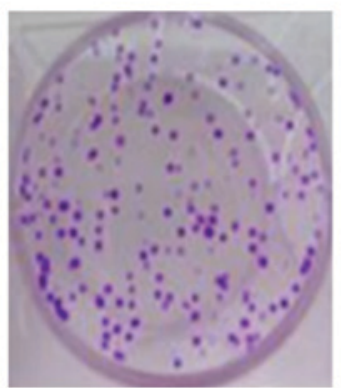

Transfection

group

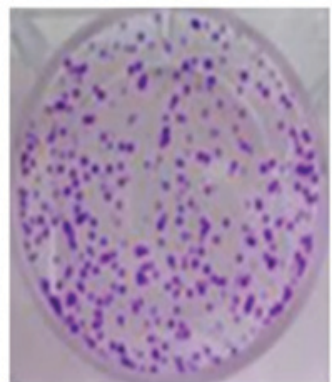

Saos2 group

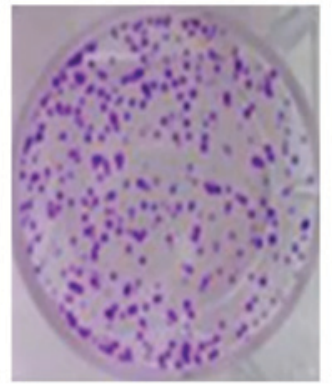

No-load transfection group
Figure 2. Cell growth in each group.

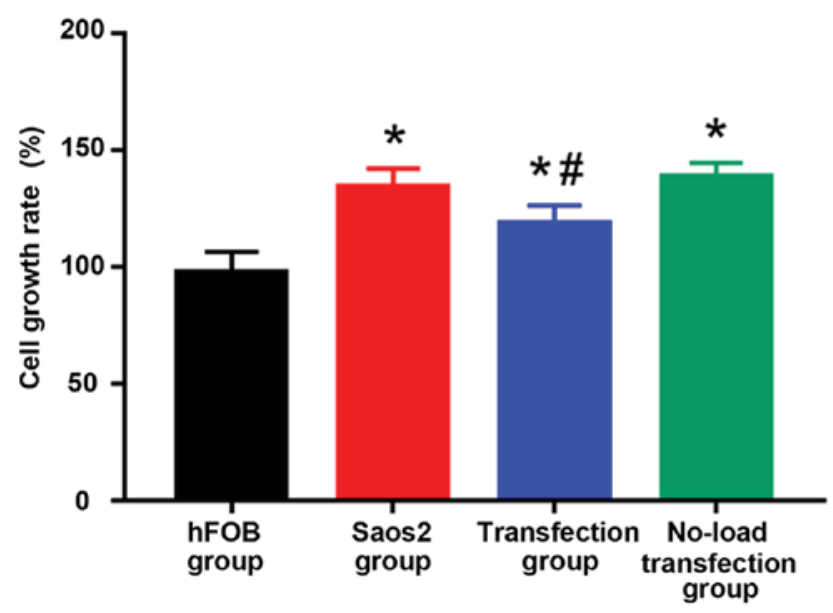

Figure 3. Cell proliferation rate in each group. ${ }^{*} \mathrm{P}<0.05$ vs. hFOB group; ${ }^{\text {"P }}<0.05$ vs. Saos 2 group.

FHIT mRNA is highly expressed in normal osteoblast cell line, but lowly expressed in Saos 2 osteosarcoma cell line. The transfection of pcDNA3.1-FHIT overexpression vectors containing FHIT gene into Saos 2 cells can promote the expression of FHIT mRNA, indicating that the transfection method is effective.

Detection of cell proliferation by CCK8 assay. Cell growth is shown in Fig. 2 hFOB group had moderate cell density, Saos2 group and no-load transfection group had high cell density with concentrated cells. The cell density in the transfection group was lower than that in Saos2 and no-load transfection groups. CCK8 results are shown in Fig. 3: Compared with that in hFOB group, cell proliferation was significantly increased in the other groups, and the differences were 

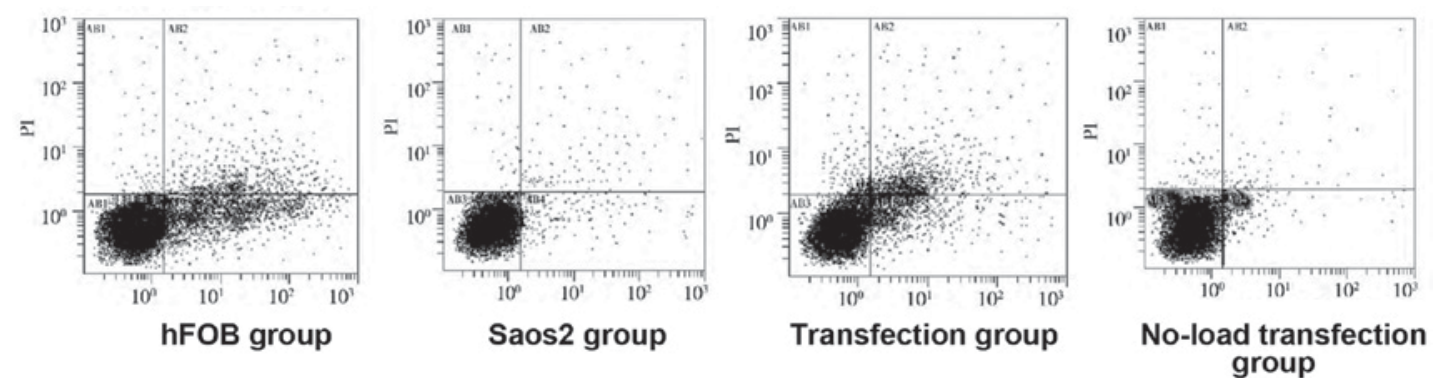

Figure 4. Detection of apoptosis by flow cytometry.

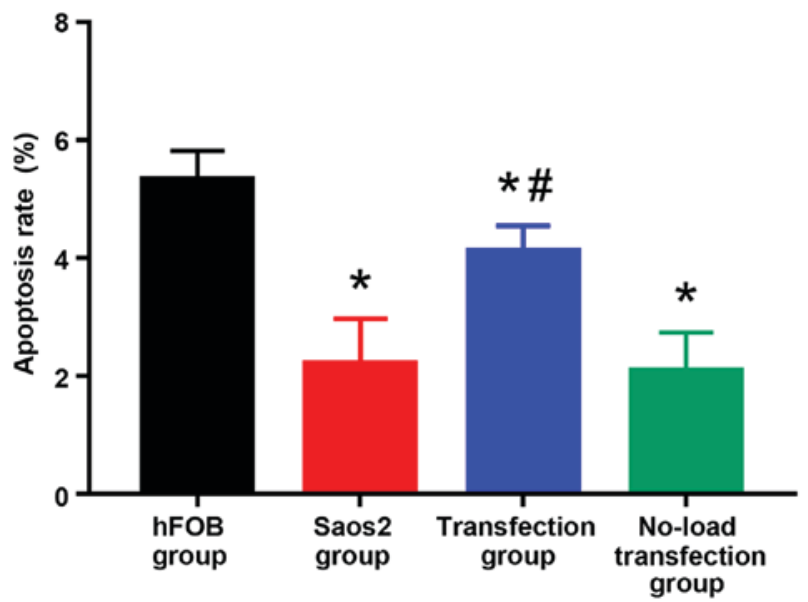

Figure 5. Apoptosis rate in each group. ${ }^{*} \mathrm{P}<0.05$ vs. $\mathrm{hFOB}$ group; ${ }^{\#} \mathrm{P}<0.05$ vs. Saos2 group.

statistically significant $(\mathrm{P}<0.05)$. Compared with that in Saos2 group, cell proliferation was significantly decreased in transfection group, and the difference was statistically significant $(\mathrm{P}<0.05)$. The results suggested that the proliferation of normal osteoblast cell line is lower, and that of Saos2 osteosarcoma cell line is higher. The transfection of pcDNA3.1-FHIT overexpression vectors containing FHIT gene into Saos 2 cells can inhibit the proliferation of Saos2 osteosarcoma cells.

Detection of apoptosis by flow cytometry. As shown in Figs. 4 and 5, compared with that in hFOB group, the apoptosis rates in the other groups were significantly decreased, and there were statistically significant differences $(\mathrm{P}<0.05)$. Compared with that in Saos2 group, the apoptosis rate in transfection group was significantly increased, and the difference was statistically significant $(\mathrm{P}<0.05)$. These results indicated that the apoptosis rate of normal osteoblast cell line is higher, and that of Saos2 osteosarcoma cell line is lower. The transfection of pcDNA3.1-FHIT overexpression vectors containing FHIT gene into Saos2 cells can promote apoptosis of Saos2 osteosarcoma cells.

Detection of FHIT protein expression via western blot analysis. As shown in Figs. 6 and 7, the highest expression of FHIT protein was found in hFOB group and the lowest expression was in Saos2 group. Compared with that in $\mathrm{hFOB}$ group, the expressions of FHIT protein in the other groups

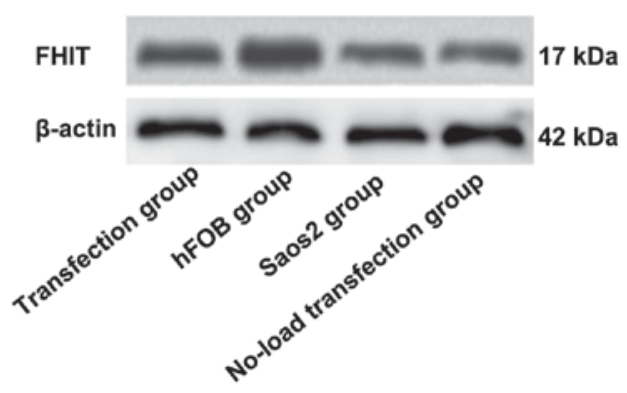

Figure 6. Detection of FHIT protein expression in each group via western blot analysis. FHIT, fragile histidine triad.

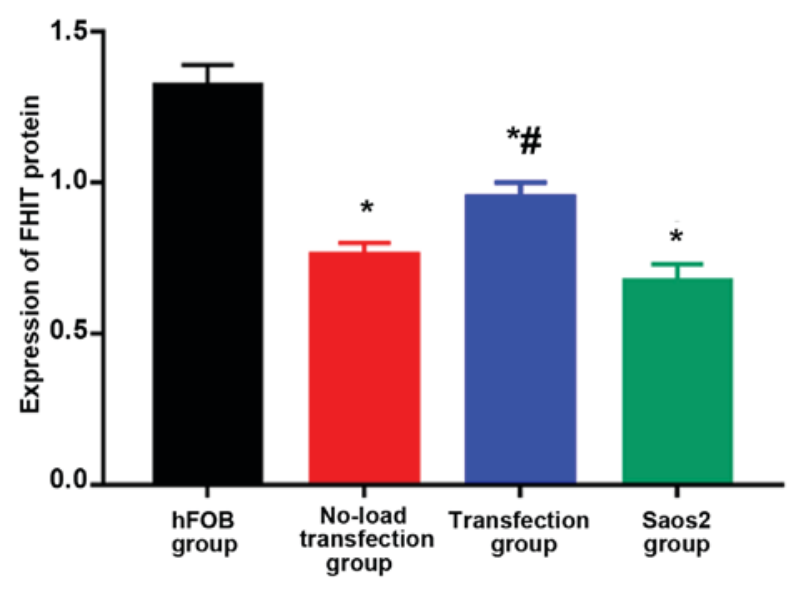

Figure 7. Relative expression of FHIT protein in each group. ${ }^{*} \mathrm{P}<0.05$ vs hFOB group; ${ }^{\text {P }}<0.05$ vs. Saos 2 group. FHIT, fragile histidine triad.

were significantly decreased, and the differences were statistically significant $(\mathrm{P}<0.05)$. Compared with that in Saos2 group, the expression of FHIT protein in transfection group was significantly increased, and there was a statistically significant difference $(\mathrm{P}<0.05)$. The results indicated that FHIT protein is highly expressed in normal osteoblast cell line, but lowly expressed in Saos2 osteosarcoma cell line. The transfection of pcDNA3.1-FHIT overexpression vectors containing FHIT gene into Saos2 cells can promote FHIT protein expression.

\section{Discussion}

Osteosarcoma is a common tumor with a high degree of malignancy, mainly derived from mesenchymal tissues (6). Osteosarcoma mainly occurs in adolescents and the growth 
is rapid, the metastasis and mortality rates are high, and the prognosis is poor $(7,8)$. With the progress of society and the development of science and technology, some achievements have been made in the treatment of osteosarcoma, and the 5 -year survival rate is about 50-60\% (9). However, there are still no effective and ideal methods for the treatment of osteosarcoma. At present, it is believed that abnormal expression and functional changes of multiple genes are important reasons for the rapid development of osteosarcoma (10). The FHIT gene is located on human chromosome 3p14.2 and encodes a protein with $16.8 \mathrm{kDa}$ in size. The protein plays an important regulatory role in the process of DNA repair and cell cycle. It is currently believed that the FHIT gene is closely related to the occurrence of many tumors. A study found that retransfection of FHIT gene in esophageal, lung and breast cancer cell lines with FHIT gene knockout can effectively inhibit the growth of cancer cells (11). As an expression product of FHIT gene, FHIT protein can significantly inhibit the growth of human colon cancer cell line and promote its apoptosis (12). Therefore, FHIT gene is considered to be an effective tumor suppressor gene. At the same time, there is a close correlation between FHIT gene and apoptosis. Transfection of FHIT gene into lung cancer cells lacking FHIT gene can promote apoptosis of lung cancer cells and block the cell cycle at G0/G1 phase (13). In addition, adenovirus vector-mediated FHIT gene plays an important role in regulating the growth, proliferation, apoptosis and long cell cycle of human tumor cells and nude mouse tumor cells. Overexpression of adenovirus vector-mediated FHIT gene in tumor cells can effectively inhibit the growth of tumor cells, but has no obvious effects on normal cells (14). At the same time, overexpression of adenovirus vector-mediated FHIT gene in tumor cells can effectively promote apoptosis of tumor cells, change the process of cell cycle and increase the number of apoptotic tumor cells $(15,16)$. Overexpressed FHIT gene and its expression product FHIT protein can be obtained by injecting adenovirus vector-mediated FHIT gene into subcutaneous tumor of nude mice, and tumor growth can be inhibited at the same time, indicating that adenovirus vector-mediated FHIT gene can be highly expressed in tumor tissues and inhibit tumor growth $(17,18)$. Therefore, FHIT gene is involved in the regulation of cell apoptosis and cell cycle process. The inhibitory effect of FHIT gene on tumor growth may be achieved by promoting apoptosis of tumor cells and inhibiting the growth of tumor cells $(19,20)$. In this study, it was found that FHIT gene was abnormally expressed in Saos2 osteosarcoma cell line, and the FHIT gene expression was suppressed in Saos2 osteosarcoma cell line compared with that in normal osteoblast cell line, including transcription and translation. Plasmid vectors carrying target gene fragments are transfected into cells to realize the stable expression of products of target genes in cells. This cell transfection method is reliable and stable, and is widely used in the cell transfection technology. In this study, plasmid vectors carrying FHIT gene were transfected into Saos 2 cells. It was found that the transfected FHIT gene could be stably expressed in Saos 2 cells, which increased the expression of FHIT gene mRNA and protein, indicating that the cell transfection was successful. At the same time, this study manifested that Saos2 osteosarcoma cell line had a high proliferation rate and a low apoptosis rate compared with normal osteoblast cell line. However, after transfection with FHIT gene, the proliferation rate of the transfected Saos2 osteosarcoma cells was decreased and the apoptosis rate was increased. These results suggest that FHIT gene regulates the proliferation and apoptosis of Saos2 osteosarcoma cells, inhibits the proliferation and promotes apoptosis of osteosarcoma cells.

\section{Acknowledgements}

Not applicable.

\section{Funding}

The study was supported by the Shanghai Medical Key Specialty Construction Fund (ZK2015A14), the Seed Fund Program of Shanghai University of Medicine \& Health Sciences (HMSF-17-22-040), the Construction of Key Discipline Group of Sanitary System of Shanghai Pudong New District (PWZxq2017-12), and the Construction of 'the most important' Discipine of Zhoupu Hospital of Shanghai Pudong New District (ZP-XK-2015a-2).

\section{Availability of data and materials}

The datasets used and/or analyzed during the current study are available from the corresponding author on reasonable request.

\section{Authors' contributions}

$\mathrm{ZX}$ wrote the manuscript and was responsible for the cell culture. JW assisted with cell treatment and transfection. PC and XZ performed western blot analysis and RT-qPCR. CY was in charge of CCK8. BW contributed to flow cytometry. All authors read and approved the final manuscript.

\section{Ethics approval and consent to participate}

The study was approved by the Ethics Committee of Zhoupu Hospital affiliated to Shanghai University of Medicine and Health Science (Shanghai, China).

\section{Patient consent for publication}

Not applicable.

\section{Competing interests}

The authors declare that they have no competing interests.

\section{References}

1. Novello C, Pazzaglia L, Cingolani C, Conti A, Quattrini I, Manara MC, Tognon M, Picci P and Benassi MS: miRNA expression profile in human osteosarcoma: Role of miR-1 and miR-133b in proliferation and cell cycle control. Int J Oncol 42: 667-675, 2013 .

2. Tang J, Shen L, Yang Q and Zhang C: Overexpression of metadherin mediates metastasis of osteosarcoma by regulating epithelial-mesenchymal transition. Cell Prolif 47: 427-434, 2014.

3. Díaz-Rodríguez L, García-Martínez O, Morales MA, Rodríguez-Pérez L, Rubio-Ruiz B and Ruiz C: Effects of indomethacin, nimesulide, and diclofenac on human MG-63 osteosarcoma cell line. Biol Res Nurs 14: 98-107, 2012. 
4. Sard L, Accornero P, Tornielli S, Delia D, Bunone G, Campiglio M, Colombo MP, Gramegna M, Croce CM, Pierotti MA, et al: The tumor-suppressor gene FHIT is involved in the regulation of apoptosis and in cell cycle control. Proc Natl Acad Sci USA 96: 8489-8492, 1999.

5. Ji L, Fang B, Yen N, Fong K, Minna JD and Roth JA: Induction of apoptosis and inhibition of tumorigenicity and tumor growth by adenovirus vector-mediated fragile histidine triad (FHIT) gene overexpression. Cancer Res 59: 3333-3339, 1999.

6. Ta HT, Dass CR, Choong PF and Dunstan DE: Osteosarcoma treatment: State of the art. Cancer Metastasis Rev 28: 247-263, 2009.

7. Sampo M, Koivikko M, Taskinen M, Kallio P, Kivioja A, Tarkkanen $\mathrm{M}$ and Böhling T: Incidence, epidemiology and treatment results of osteosarcoma in Finland - a nationwide population-based study. Acta Oncol 50: 1206-1214, 2011.

8. Mirabello L, Troisi RJ and Savage SA: Osteosarcoma incidence and survival rates from 1973 to 2004: Data from the Surveillance, Epidemiology, and End Results Program. Cancer 115: 1531-1543, 2009.

9. Jaffe N: Osteosarcoma: Review of the past, impact on the future. The American experience. Cancer Treat Res 152: 239-262, 2009.

10. Strauss SJ,Ng T, Mendoza-Naranjo A, Whelan J and Sorensen PH: Understanding micrometastatic disease and Anoikis resistance in Ewing family of tumors and osteosarcoma. Oncologist 15: 627-635, 2010.

11. Zuo H, Chan GP, Zhu J, Yeung WW, Chan AS, Ammer H and Wong YH: Activation state-dependent interaction between Gaq subunits and the Fhit tumor suppressor. Cell Commun Signal 11: 59, 2013.

12. Morikawa H, Nakagawa Y, Hashimoto K, Niki M, Egashira Y, Hirata I, Katsu K and Akao Y: Frequent altered expression of fragile histidine triad protein in human colorectal adenomas. Biochem Biophys Res Commun 278: 205-210, 2000.

13. Hu B, Ying X, Wang J, Piriyapongsa J, Jordan IK, Sheng J, Yu F, Zhao $\mathrm{P}, \mathrm{Li} \mathrm{Y}$, Wang $\mathrm{H}$, et al: Identification of a tumor-suppressive human-specific microRNA within the FHIT tumor-suppressor gene. Cancer Res 74: 2283-2294, 2014.
14. Siprashvili Z, Sozzi G, Barnes LD, McCue P, Robinson AK, Eryomin V, Sard L, Tagliabue E, Greco A, Fusetti L, et al: Replacement of Fhit in cancer cells suppresses tumorigenicity. Proc Natl Acad Sci USA 94: 13771-13776, 1997.

15. Garinis GA, Gorgoulis VG, Mariatos G, Zacharatos P, Kotsinas A, Liloglou T, Foukas P, Kanavaros P, Kastrinakis NG, Vassilakopoulos T, et al: Association of allelic loss at the FHIT locus and p53 alterations with tumour kinetics and chromosomal instability in non-small cell lung carcinomas (NSCLCs). J Pathol 193: 55-65, 2001.

16. Lin HY, Hung SK, Lee MS, Chiou WY, Huang TT, Tseng CE, Shih LY, Lin RI, Lin JM, Lai YH, et al: DNA methylome analysis identifies epigenetic silencing of FHIT as a determining factor for radiosensitivity in oral cancer: An outcome-predicting and treatment-implicating study. Oncotarget 6: 915-934, 2015.

17. Gemma A, Hagiwara K, Ke Y, Burke LM, Khan MA, Nagashima M, Bennett WP and Harris CC: FHIT mutations in human primary gastric cancer. Cancer Res 57: 1435-1437, 1997.

18. Sevignani C, Calin GA, Cesari R, Sarti M, Ishii H, Yendamuri S, Vecchione A, Trapasso F and Croce CM: Restoration of fragile histidine triad (FHIT) expression induces apoptosis and suppresses tumorigenicity in breast cancer cell lines. Cancer Res 63: 1183-1187, 2003.

19. Fang JM, Arlt MF, Burgess AC, Dagenais SL, Beer DG and Glover TW: Translocation breakpoints in FHIT and FRA3B in both homologs of chromosome 3 in an esophageal adenocarcinoma. Genes Chromosomes Cancer 30: 292-298, 2001.

20. Huiping C, Jonasson JG, Agnarsson BA, Sigbjornsdottir BI, Huebner $\mathrm{K}$ and Ingvarsson $\mathrm{S}$ : Analysis of the fragile histidine triad (FHIT) gene in lobular breast cancer. Eur J Cancer 36: $1552-1557,2000$

This work is licensed under a Creative Commons Attribution-NonCommercial-NoDerivatives 4.0 International (CC BY-NC-ND 4.0) License. 